Research Paper

\title{
Circular RNA CircHIPK3 Promotes Gemcitabine Sensitivity in Bladder Cancer
}

\author{
Fang Xie ${ }^{1}$, Ning Zhao ${ }^{2}$, Hui Zhang ${ }^{3}$, Dalong Xie ${ }^{4}$ \\ 1. Medical Basic Experimental Teaching Center, China Medical University, Shenyang, 110122, China \\ 2. Surgery Laboratory, Affiliated First Hospital, China Medical University, Shenyang, 110001, China \\ 3. Department of Urinary surgery, Shengjing Hospital, China Medical University, Shenyang, 110004, China \\ 4. Department of Anatomy, College of Basic Medicine, China Medical University, Shenyang, 110122, China \\ $\triangle$ Corresponding author: Dalong Xie, Department of Anatomy, College of Basic Medicine, China Medical University, No.77 Puhe Road, Shenyang North New \\ Area, Shenyang, 110122, China. Phone/Fax: +86-24-31939094; E-mail address: dlxie@cmu.edu.cn \\ (C) The author(s). This is an open access article distributed under the terms of the Creative Commons Attribution License (https://creativecommons.org/licenses/by/4.0/). \\ See http://ivyspring.com/terms for full terms and conditions.
}

Received: 2019.08.28; Accepted: 2019.12.22; Published: 2020.01.22

\begin{abstract}
Purpose: Recent studies showed circular RNA (circRNA) played important regulatory roles in tumors, including genesis of chemotherapy resistance. In this study, the role of circHIPK 3 on chemotherapy resistance of bladder cancer $(B C)$ will be clarified.

Methods: Real-time quantitative PCR was applied to examine the circHIPK3 expression. The gemcitabine sensitivity and cell proliferation viability were analyzed by Cell Counting Kit- 8 assay. Double-stained flow cytometry was used to detect the cell apoptosis.

Results: In BC tissues and cell lines, the circHIPK3 expression was down-regulated. Its expression had a negative correlation with pathological grade, lymph node metastasis and gemcitabine insensitivity of BC patients. CircHIPK3 was a independent prognostic biomarker for BC patients. The expression of circHIPK3 in T24/gem and J82/gem cell lines (resistant to gemcitabine) was down-regulated significantly. The over-expression of circHIPK3 decreased IC50 of gemcitabine and promoted gemcitabine's cytotoxicity in T24/gem and J82/gem cells.

Conclusions: The circHIPK3 is low-expressed in BC and is an independent prognostic biomarker for BC patients. The low-expression of circHIPK3 is associated with the insensitivity to gemcitabine of $B C$ patients, over-expression of circHIPK3 promotes gemcitabine sensitivity in BC.
\end{abstract}

Key words: bladder cancer; circular RNA; circHIPK3; chemotherapy; gemcitabine

\section{Introduction}

Bladder cancer $(\mathrm{BC})$ is one of the ten most common tumors in the whole body and the most common malignant tumors in urinary system [1]. In the West, the incidence of $\mathrm{BC}$ is second only to prostate cancer in urogenital tumors, while it occupies the first place in China [1-3]. Bladder urothelial carcinoma is the most common pathological type of $\mathrm{BC}$, accounting for more than $90 \%$ of the total number of $\mathrm{BC}$ patients.

Chemotherapy is one of the most effective means to treat $\mathrm{BC}$ at present, which reduced significantly the risk of recurrence and metastasis, and improve evidently the prognosis of BC patients [4]. Nevertheless, the resistance of cancer cells to chemotherapy drugs often leads to chemotherapy failure.

Recently, circular RNA (circRNA) is a hotspot in the field of life science research, it is a special kind of non-coding RNA molecule. Because the closed ring structure is not affected by RNA exonuclease, circRNAs are more stable and difficult to degrade. CircRNA molecules are rich in microRNA binding sites and act as competitive endogenous RNA (ceRNA) to play the role of microRNA sponge in cells $[5,6]$. By interacting with tumor-associated microRNAs, circRNAs play important regulatory roles in tumorigenesis [7-10]. 
CircHIPK3 (circRNA ID: hsa_circ_0000284), also named bladder cancer-related circular RNA-2 (BCRC-2), originates from the second exon of Homeodomain-interacting protein kinase 3 (HIPK3) gene and consists of 1099 nucleotides in length. Recent studies confirmed that circHIPK3 showed the abnormal expression level and functions in various tumors, included prostate cancer, gastric cancer, lung cancer, ovarian cancer, and so on [11-16]. In addition, circHIPK3 contributed to lung fibroblast-tomyofibroblast transition in pulmonary fibrosis [17], and promoted the proliferation and migration of cardiac fibroblasts in cardiac fibrosis [18].

CircHIPK3 was low-expressed in BC, up-regulation of circHIPK3 suppressed the angiogenesis, invasion, and migration of $\mathrm{BC}$ cells, and inhibited the growth and metastasis of $B C$ in vivo [19]. In our preliminary experiment, circRNA microarray screening found that circHIPK3 was also down-regulated in BC tissues, which suggested circHIPK3 might be involved in the genesis of $\mathrm{BC}$. Nevertheless, it is not clear whether circHIPK3 is involved in the formation of chemotherapy resistance in $\mathrm{BC}$ and its role in chemotherapy resistance.

\section{Methods}

\section{Clinic specimens}

$68 \mathrm{BC}$ tissue and corresponding normal bladder urothelial tissue (NBUT) specimens were obtained Shengjing Hospital from August 2012 to April 2014. All tissue specimens were collected through cystoscopy and confirmed by pathological diagnosis. All patients received no chemotherapy, radiotherapy or other antineoplastic treatment before diagnosis. The inclusion criteria of patients was that all patients were diagnosed as BC, and they needed neoadjuvant chemotherapy with gemcitabine before operation or they chose gemcitabine chemotherapy if they could not be treated surgically. The exclusion criteria was the BC patient did not treat with gemcitabine chemotherapy.

After definite diagnosis, all patients received gemcitabine treatment. The curative effects are verified according to cystoscopy and enhancement CT scanning after three cycles of chemotherapy. All patients were divided into sensitive group $(n=38)$ and insensitive group $(\mathrm{n}=30)$ according to the therapeutic effects.

\section{Cell lines and culture}

Human fetal bladder tissue derived CCC-HB-2 cell line, human bladder epithelial immortalized cell line SV-HUC-1 and BC cell lines (T24, J82, UMUC3) were stored in our laboratory and identified by STR Authentication. T24/gem and J82/gem cell lines (resistant to gemcitabine) were established in previous studies $[20,21]$. All cells were cultured in DMEM medium with 10\% fetal bovine serum (ExCell Bio, Shanghai, USA) in a $37^{\circ} \mathrm{C}$ incubator.

\section{Real-time quantitative PCR}

The extraction, purification and identification of RNA were applied all according to our previous studies [20,21]. The A260/A280 ratio of purified RNA was typically between 1.8 and 2.4 and the yield between $80 \mu \mathrm{g}$ and $120 \mu \mathrm{g}$. RNA samples were stored at $-80^{\circ} \mathrm{C}$. RNA integrity was assessed by gel electrophoresis. RNase $\mathrm{R}$ was used to eliminate the linear RNAs. One Step SYBR RT-PCR Kit (Takara, Dalian, Liaoning, China) was used to qualify the expression level of circHIPK3 according to manufacturer's instructions using a 7700 PCR System (Applied Biosystems, ThermoFisher, Foster City, CA, USA). Each reaction contained $2 \times$ One Step SYBR RT-PCR Buffer $(12.5 \mu \mathrm{L})$, PrimeScript 1 Step Enzyme Mix $2(1 \mu \mathrm{L})$, Forward Primer $(10 \mu \mathrm{M}, 1 \mu \mathrm{L})$, Reverse Primer $(10 \mu \mathrm{M}, 1.25 \mu \mathrm{L})$, RNA template $(2 \mu \mathrm{L})$ and RNase Free $\mathrm{dH} 2 \mathrm{O}(7.5 \mu \mathrm{L})$. The cycling condition is as follows: Stage 1: $42^{\circ} \mathrm{C}$ for $5 \mathrm{~min}, 95^{\circ} \mathrm{C}$ for $10 \mathrm{sec}, 1$ Cycle; Stage 2: $95^{\circ} \mathrm{C}$ for $5 \mathrm{sec}, 60^{\circ} \mathrm{C}$ for $30 \mathrm{sec}, 40$ Cycles; Stage 3: $95^{\circ} \mathrm{C}$ for $15 \mathrm{sec}, 60^{\circ} \mathrm{C}$ for $30 \mathrm{sec}, 95^{\circ} \mathrm{C}$ for $15 \mathrm{sec}, 1$ Cycles. The primers for circHIPK3 were 5'- TTCAACATATCTACAATCTCGGT -3' (sense) and 5' - ACCATTCACATAGGTCCGT -3' (antisense) [19]. After normalization with reference to expression of GAPDH, the relative expression level of circHIPK3 was calculated as $2^{-\Delta \Delta C T}$ method.

\section{Cells Transfection}

The expression plasmid and negative control plasmid of circHIPK3 (pCD5-circHIPK3 and pCD5-NC) were constructed by GENESEED Company (Guangzhou, Guangdong, China). Lipofectamine $^{\mathrm{TM}} 3000$ (Thermo Fisher Scientific, USA) was applied to transfect plasmids into cells according to manufacturer's instructions.

\section{Cell viability detection}

Cell Counting Kit-8 (MedChemExpress, Monmouth Junction, NJ, USA) was used to examine cell viability. $5 \times 10^{3}$ cells were suspended with $100 \mu \mathrm{l}$ culture medium and seed in a well of a 96-well plate; $10 \mu \mathrm{l} \mathrm{CCK-8} \mathrm{solution} \mathrm{was} \mathrm{added} \mathrm{to} \mathrm{each} \mathrm{well} \mathrm{of} \mathrm{the}$ plate; the plate was incubated $1 \mathrm{~h}$ in the incubator and mix gently on an orbital shaker for $1 \mathrm{~min}$. The absorbance at $450 \mathrm{~nm}$ was measured using a microplate reader.

\section{Gemcitabine sensitivity detection}

$5 \times 10^{3}$ cells were suspended with $100 \mu$ l culture medium and seed in a well of a 96-well plate; new 
culture medium with gemcitabine $(0.1 \mu \mathrm{g} / \mathrm{ml}, 0.5$ $\mu \mathrm{g} / \mathrm{ml}, 1 \mu \mathrm{g} / \mathrm{ml}, 5 \mu \mathrm{g} / \mathrm{ml}$ and $10 \mu \mathrm{g} / \mathrm{ml}$ respectively) (Sigma-Aldrich, St. Louis, MO, USA) was added to replace old culture medium $6 \mathrm{~h}$ later; after $24 \mathrm{~h}$, cell viability was examined and the half maximal inhibitory concentration (IC50) was calculated according to our previous studies $[20,21]$.

\section{Cell apoptosis detection}

Annexin V-FITC apoptosis detection kit (Meilunbio, Dalian, Liaoning, China) was applied to detect cell apoptosis rate. $1 \times 10^{5}$ cells were suspend in $100 \mu \mathrm{l}$ culture medium and added $5 \mu \mathrm{l}$ Annexin V-FITC and $5 \mu \mathrm{PI}$; cell suspension incubated at room temperature and without light for $15 \mathrm{~min} ; 400 \mu \mathrm{l}$ Binding Buffer was added and and mixed gently; then cell apoptosis rate was examined and analyzed according to our previous studies $[20,21]$.

\section{Statistical analysis}

All data was analyzed using Graphpad prism 5 (GraphPad Software, San Diego, CA, USA). All BC patients were divided into two groups (high-expression and low-expression) according to the median value of relative circHIPK3 expression. The difference comparison was analyzed by one-way ANOVA, Student's t-test and Chi-square test. The survival rate was calculated with Kaplan-Meier method with the log-rank test for comparisons.

Variables with a value of $P<0.05$ in the univariate analysis were included in the subsequent multivariate analysis based on the Cox proportional hazards model. A $P$ value of less than 0.05 was considered to be statistically significant.

\section{Results}

\section{The expression of circHIPK 3 was down-regulated in BC}

Firstly, the circRNAs which differentially expressed between BC and NBUT were screened using circRNA microarray assay, screening results suggest that the expression of circHIPK3 had a prominent down-regulation in $\mathrm{BC}$ compared with NBUT (Figure 1A). Secondly, real-time quantitative PCR confirmed circHIPK3 was significantly low-expressed in BC in comparison to NBUT (Figure $1 \mathrm{~B}, P<0.05)$. Thirdly, the circHIPK3 expression in T24, J82 and UMUC3 cells was much lower than that in CCC-HB-2 and SV-HUC-1 cells (Figure $1 C, P<0.05$ ).

As shown in Table 1, the circHIPK3 expression had a negative correlation with high pathological grade, lymph node metastasis and gemcitabine chemotherapy insensitivity of $\mathrm{BC}$ patients $(P<0.05)$, but was not correlated with age, gender, smoking history and muscular invasion of $\mathrm{BC}$ patients $(P>0.05)$.

\section{B}

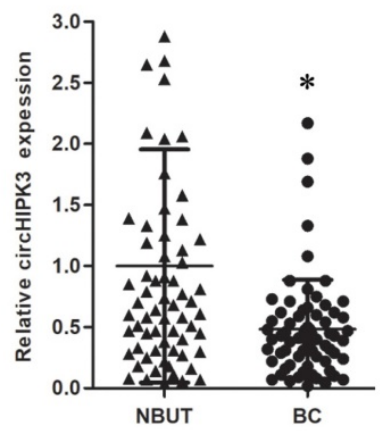

C

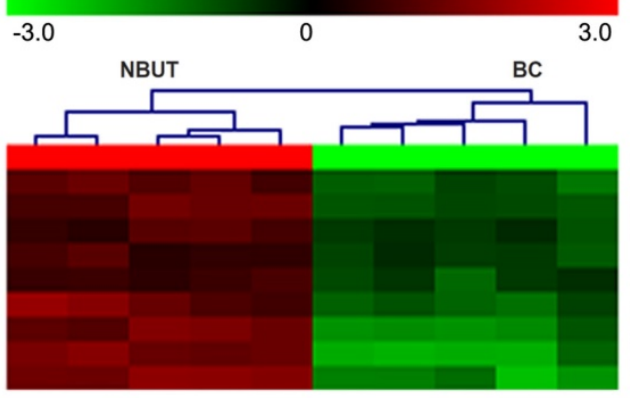

hsa_circ_0018064 hsa_circ_0017636 hsa_circ_0000284 hsa_circ_0004119 hsa_circ_0042103 hsa_circ_000604 hsa circ 0004689 hsa circ 0002387 hsa_circ_0005406 hsa_circ_0081963 NBUT $\mathrm{BC}$

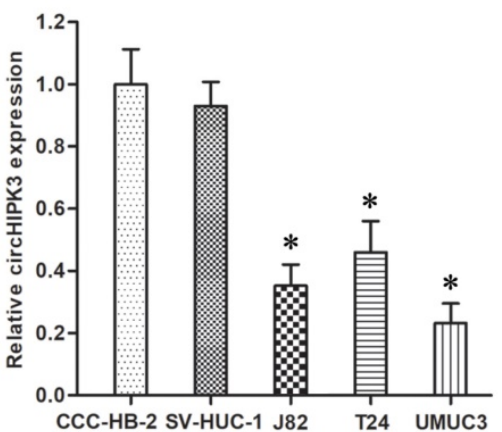

D

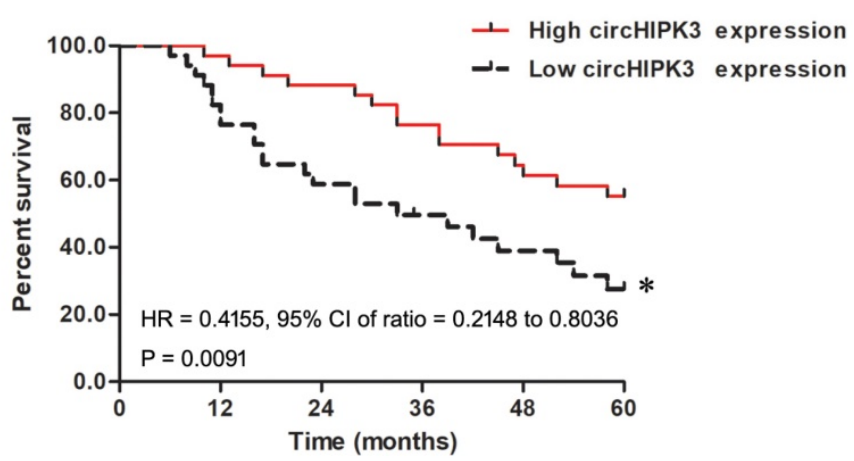

Figure 1: The expression of circHIPK3 was down-regulated in BC. A: Representative down-regulated circRNAs in BC compared with NBUT analyzed by microarray assay $(n=5)$. B: The expression of circHIPK 3 in BC was down-regulated compared with NBUT ( $n=68)$. $*$ P<0.05 vs. NBUT. C: The expression of circHIPK3 in T24, J82 and UMUC 3 cells was down-regulated in comparison with CCC-HB-2 and SV-HUC-1 cells. $* P<0.05$ vs. CCC-HB-2 and SV-HUC-1 cells. D: Kaplan-Meier analysis indicated that BC patients with lower circHIPK 3 expression exhibited a shorter survival time than those with higher expression. ${ }^{*} P<0.05$ vs. Low circHIPK 3 expression group. 
Table 1. The association of the circHIPK3 expression and clinical pathological factors of $68 \mathrm{BC}$ patients

\begin{tabular}{|c|c|c|c|c|c|}
\hline \multirow[t]{2}{*}{ Factors } & & \multicolumn{4}{|c|}{ Relative circHIPK3 expression } \\
\hline & & Number & High & Low & $P$ value \\
\hline \multirow[t]{2}{*}{ Age } & $<55$ & 34 & 15 & 19 & 0.332 \\
\hline & $\geq 55$ & 34 & 19 & 15 & \\
\hline \multirow[t]{2}{*}{ Gender } & Male & 39 & 21 & 18 & 0.462 \\
\hline & Female & 29 & 13 & 16 & \\
\hline \multirow{2}{*}{$\begin{array}{l}\text { Smoking } \\
\text { history } \\
\text { (more than ten } \\
\text { years) }\end{array}$} & No & 42 & 24 & 18 & 0.134 \\
\hline & Yes & 26 & 10 & 16 & \\
\hline \multirow[t]{2}{*}{ Grade } & Low grade & 36 & 23 & 13 & $0.015^{*}$ \\
\hline & High grade & 32 & 11 & 21 & \\
\hline \multirow{2}{*}{$\begin{array}{l}\text { Muscular } \\
\text { invasion }\end{array}$} & No & 38 & 24 & 14 & 0.052 \\
\hline & Yes & 30 & 10 & 20 & \\
\hline \multirow{2}{*}{$\begin{array}{l}\text { Lymph node } \\
\text { metastasis }\end{array}$} & No & 43 & 26 & 17 & $0.024^{*}$ \\
\hline & Yes & 25 & 8 & 17 & \\
\hline \multirow{2}{*}{$\begin{array}{l}\text { Gemcitabine } \\
\text { chemotherapy }\end{array}$} & Sensitive & 38 & 25 & 13 & $0.003^{*}$ \\
\hline & Insensitive & 30 & 9 & 21 & \\
\hline
\end{tabular}

Chi-square test, ${ }^{*} P<0.05$

Table 2. The influence of the circHIPK 3 expression and clinical characteristics on DFS in BC patients

\begin{tabular}{lllllll}
\hline \multirow{2}{*}{ Factors } & \multicolumn{3}{l}{ Univariate analysis } & \multicolumn{3}{l}{ Multivariate analysis } \\
\cline { 2 - 6 } & HR & $95 \%$ CI & $P$ value & HR & $95 \%$ CI & $P$ value \\
\hline $\begin{array}{l}\text { The circhipk3 } \\
\text { expression }\end{array}$ & 4.315 & $1.597-8.221$ & $0.006^{*}$ & 3.364 & $1.297-8.768$ & $0.007^{*}$ \\
$\begin{array}{l}\text { Grade } \\
\text { Lymph node }\end{array}$ & 2.202 & $1.035-2.395$ & 0.043 & & & \\
metastasis & 0.276 & $0.035-1.288$ & 0.076 & & & \\
$* P<0.05$ & & & & & & \\
& & & & & & \\
\end{tabular}

\section{circHIPK3 was an independent prognostic biomarker for BC patients}

The 5 year disease free survival rate (DFS) in lower or higher circHIPK3 expression group were $32.3 \%$ and $55.9 \%$ respectively, the Kaplan-Meier analysis found BC patients with lower circHIPK3 expression exhibited a shorter survival time than those with higher circHIPK3 expression (Figure 1D, $P<0.05)$. Moreover, Cox regression analysis showed that lower circHIPK3 expression was an independent prognostic biomarker for DPS in BC patients (Table 2).

\section{Low-expression of circHIPK3 correlated to gemcitabine resistance in $\mathrm{BC}$}

In consideration of the negative correlation between the circHIPK3 expression and gemcitabine insensitivity of BC chemotherapy, the circHIPK3 might participate in the chemotherapy resistance of BC. In order to test this hypothesis, the circHIPK3 expression was examined in gemcitabine-resistant cell lines.

The results showed the circHIPK3 expression in T24/gem and J82/gem cell lines was significantly down-regulated as compared to their parental cells in vitro (Figure 2, $P<0.05$ ). This data suggested that low-expression of circHIPK3 correlated to gemcitabine resistance in $\mathrm{BC}$.

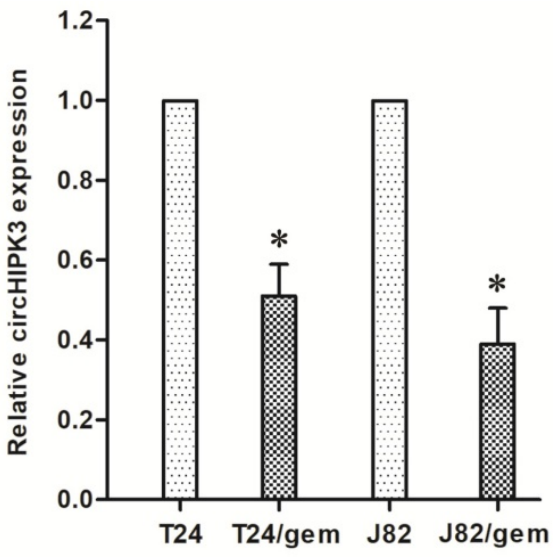

Figure 2. Compared with T24 and $\mathrm{J} 82$ cells, the expression of circHIPK3 in gemcitabine-resistant T24/gem and J82/gem cell lines was significantly down-regulated. $* P<0.05$ vs. $\mathrm{T} 24$ and $\mathrm{J} 82$ cells.

\section{Over-expression of circHIPK3 re-sensitized gemcitabine-resistant $B C$ cells to gemcitabine}

To verify the roles of circHIPK3 on chemotherapy resistance, pCD5-circHIPK3 transfected into $\mathrm{T} 24 /$ gem and $\mathrm{J} 82 /$ gem cells to up-regulated the circHIPK3 expression (Figure 3A, $P<0.05)$. And, over-expression of circHIPK3 decreased the IC50 of gemcitabine in T24/gem and J82/gem cells (Figure 3B, $P<0.05$ ), which certified that over-expression of circHIPK3 re-sensitized T24/gem and J82/gem cells to gemcitabine.

In addition, over-expression of circHIPK3 inhibited cell viability and advanced cell apoptosis (Figure 3C \& 3D, $P<0.05$ ) in T24/gem and J82/gem cells treated with $0.5 \mu \mathrm{g} / \mathrm{ml}$ gemcitabine. Over-expression of circHIPK3 significantly promoted gemcitabine's cytotoxicity in gemcitabine-resistant BC cells.

\section{Discussion}

Increasing researches found some circRNAs was involved in the genesis and progress of $\mathrm{BC}$. For instance, circMTO1 was down-regulated in BC, its expression level was negatively correlated with metastasis and poorer survival of $\mathrm{BC}$ patients [22]. $\mathrm{Lu}$ $\mathrm{Q}$ et al. reported that circSLC8A1 was low-expressed in BC, circSLC8A1 up-regulation suppressed proliferation, migration and invasion of $\mathrm{BC}$ cells [23]. Circ-0000285 was down-regulated significantly in BC tissues and serum, its expression as was associated with TNM stage, differentiation, tumor size, lymph node metastasis and distant metastasis, and the circ-0000285 expression was lower in cisplatin-resistant $\mathrm{BC}$ patients than in those who were cisplatin-sensitive, which suggested that circ-0000285 was an independent prognostic factor for the outcome of BC patient [24]. 
A

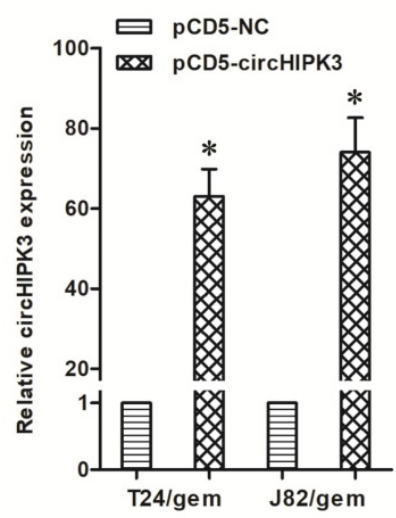

B

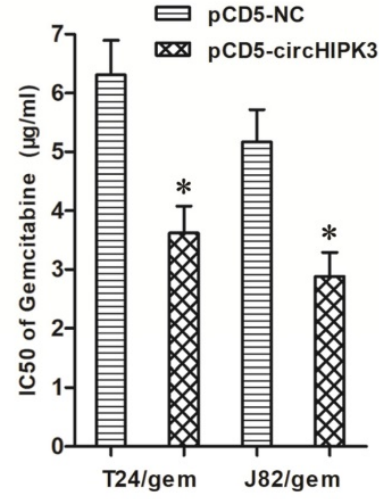

C
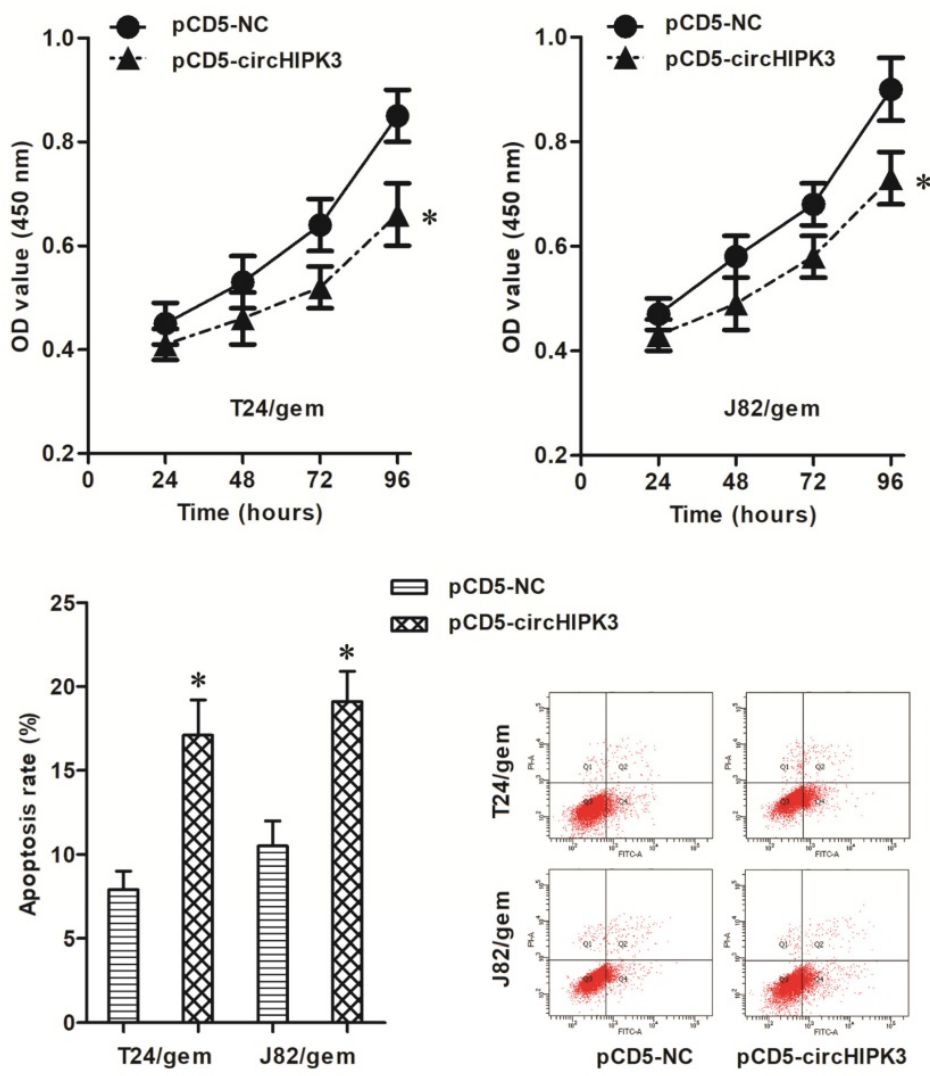

pCD5-NC

XCD5-circHIPK3
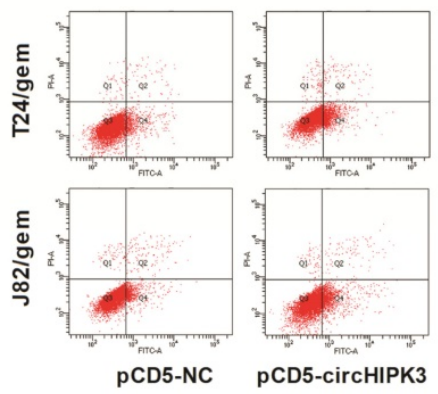

Figure 3. Up-regulation of circHIPK3 re-sensitized gemcitabine-resistant BC cells to gemcitabine. A: Transfection of $\mathrm{PCD} 5$-circHIPK3 up-regulated the expression of circHIPK3 in T24/gem and J82/gem cells. B: Over-expression of circHIPK3 decreased the IC50 of gemcitabine in T24/Gem and J82/gem cells C: Over-expression of circHIPK 3 suppressed cell proliferation in T24/Gem and J82/gem cells treated with $0.5 \mu \mathrm{g} / \mathrm{ml}$ Gemcitabine. D: Over-expression of circHIPK3 promoted apoptosis in T24/Gem and $\mathrm{J} 82 / \mathrm{gem}$ cells treating with $0.5 \mu \mathrm{g} / \mathrm{ml}$ Gemcitabine. $* P<0.05$ vs. $\mathrm{PCD} 5-\mathrm{NC}$ group.

Firstly, real-time quantitative PCR and microarray assays found circHIPK3 was low-expressed in BC tissues and cell lines, and its expression was negatively related with pathological grade and lymph node metastasis of $\mathrm{BC}$. Moreover, circHIPK3 was a independent prognostic biomarker for BC patients, patients with lower circHIPK3 expression showed a significantly worse prognosis. Thus, circHIPK3 played important roles in the genesis and progress of $\mathrm{BC}$.

$\mathrm{Li} Y$ et al. reported circHIPK3 was low-expressed in $\mathrm{BC}$, and negatively correlated with grades, invasion and lymph node metastasis of BC patients, which was consistent with our findings and further demonstrated the roles of circHIPK3 in the genesis and progression of BC [22]. However, circHIPK3 was found to be highly expressed in glioma and elevated level of circHIPK3 was linked to poor prognosis [25]. This suggested that our circHIPK3 played different roles in different malignant tumors.

In addition, the low-expression of circHIPK3 was related with insensitivity of $\mathrm{BC}$ patients to gemcitabine, which suggested circHIPK3 might be involved in chemotherapy resistance of BC. Furtherly,
circHIPK3 was down-regulated in gemcitabineresistant T24/gem and J82/gem cells compared with their parental cells. These findings preliminarily confirmed circHIPK3 took part in the genesis of chemotherapy resistance in $\mathrm{BC}$.

Therefore, the impacts of circHIPK3 on gemcitabine sensitivity in $\mathrm{BC}$ were examined through a series of gain of function assays. The results showed over-expression of circHIPK3 reduced IC50 of gemcitabine and advanced gemcitabine's cytotoxicity in gemcitabine-resistant cells, which showed over-expression of circHIPK3 re-sensitized gemcitabine-resistant $\mathrm{BC}$ cells to gemcitabine. However, the underlying mechanism is unclear.

It is well known that some circRNA molecules are rich in microRNA binding sites and can act as ceRNAs to adsorb microRNAs. For example, circAKT3 could up-regulate PIK3R1 through adsorbing miR-198, and then enhance cisplatin resistance in gastric cancer [26]; circPAN3 sponge $\mathrm{miR}-153-5 \mathrm{p} / \mathrm{miR}-183-5 \mathrm{p}$ to mediate the doxorubicin chemoresistance in acute myeloid leukemia [27]. Recently, three literatures reported circHIPK3 could sponge miR-124 in lung cancer, gallbladder cancer 
and hepatocellular carcinoma [28-30]. In addition, miR-124 regulated the gemcitabine resistance in pancreatic cancer and enhanced gemcitabine-induced cell apoptosis [31]. These findings give us a nice enlightenment, accordingly, we hypothesize circHIPK3 act as ceRNA to sponges some microRNAs and then regulate their target genes, and this mechanism is involved in genesis of chemotherapy resistance in $\mathrm{BC}$.

In conclusion, the circHIPK3 is low-expressed in $\mathrm{BC}$ and is an independent prognostic biomarker for BC patients. The low-expression of circHIPK3 is related with the gemcitabine insensitivity of $\mathrm{BC}$ patients, over-expression of circHIPK3 promotes gemcitabine sensitivity in BC. Our study helps to expound the mechanism of chemotherapy resistance in $\mathrm{BC}$, and future study may provide a novel therapeutic target for BC. However, whether our hypothesis is correct or whether there are other possible regulatory mechanisms has not been studied in this study, which needs to be further explored.

\section{Acknowledgments}

This study was funded by the National Nature Science Foundation of China (81872067, 30901480, 81301834).

\section{Author Contributions}

FX, NZ and DX carried out the experiments and performed the statistical analysis. HZ and DX participated in the study design and drafted the manuscript. All authors read and approved the final manuscript.

\section{Ethical Approval}

This study were approved by the Medical Ethics Committee of Shengiing Hospital, and all patients were informed and signed informed consent.

\section{Competing Interests}

The authors have declared that no competing interest exists.

\section{References}

1. Siegel RL, Miller KD, Jemal A. Cancer statistics, 2019. CA Cancer J Clin. 2019; 69(1):7-34.

2. Chen W, Zheng R, Baade PD, et al. Cancer statistics in China, 2015. CA Cancer J Clin. 2016; 66(2):115-32.

3. Antoni S, Ferlay J, Soerjomataram I, et al. Bladder Cancer Incidence and Mortality: A Global Overview and Recent Trends. Eur Urol. 2017; 71(1):96-108.

4. Martinez Rodriguez RH, Buisan Rueda O, Ibarz L. Bladder cancer: Present and future. Med Clin (Barc). 2017; 149(10):449-455.

5. Kristensen LS, Andersen MS, Stagsted LVW, et al. The biogenesis, biology and characterization of circular RNAs. Nat Rev Genet. 2019; 20(11):675-691.

6. Panda AC. Circular RNAs Act as miRNA Sponges. Adv Exp Med Biol. 2018; 1087:67-79.

7. Bach DH, Lee SK, Sood AK. Circular RNAs in Cancer. Mol Ther Nucleic Acids. 2019; 16:118-129.

8. Xiao-Long M, Kun-Peng Z, Chun-Lin Z. Circular RNA circ_HIPK3 is down-regulated and suppresses cell proliferation, migration and invasion in osteosarcoma. J Cancer. 2018; 9(10):1856-1862.
9. Zheng SQ, Qi Y, Wu J, et al. CircPCMTD1 Acts as the Sponge of miR-224-5p to Promote Glioma Progression. Front Oncol. 2019; 9:398.

10. Zhou JZ, Hu MR, Diao HL, et al. Comprehensive analysis of differentially expressed circRNAs revealed a ceRNA network in pancreatic ductaladenocarcinoma. Arch Med Sci. 2019; 15(4):979-991.

11. Cai C, Zhi Y, Wang K, et al. CircHIPK3 overexpression accelerates the proliferation and invasion of prostate cancer cells through regulating miRNA-338-3p. Onco Targets Ther. 2019; 12:3363-3372.

12. Chen D, Lu X, Yang F, Xing N. Circular RNA circHIPK3 promotes cell proliferation and invasion of prostate cancer by sponging miR-193a-3p and regulating MCL1 expression. Cancer Manag Res. 2019; 11:1415-1423.

13. Ghasemi S, Emadi-Baygi M, Nikpour P. Down-regulation of circular RNA ITCH and circHIPK3 in gastric cancer tissues. Turk J Med Sci. 2019; 49(2):687-695.

14. Teng F, Xu J, Zhang M, et al. Comprehensive circular RNA expression profiles and the tumor-suppressive function of circHIPK3 in ovarian cancer. Int J Biochem Cell Biol. 2019; 112:8-17.

15. Liu N, Zhang J, Zhang LY, Wang L. CircHIPK3 is upregulated and predicts a poor prognosis in epithelial ovarian cancer. Eur Rev Med Pharmacol Sci. 2018; 22(12):3713-3718

16. Ma XL, Zhu KP, Zhang CL. Circular RNA circ_HIPK3 is down-regulated and suppresses cell proliferation, migration and invasion in osteosarcoma. J Cancer. 2018; 9(10):1856-1862.

17. Zhang JX $\mathrm{Lu} \mathrm{J}$ Xie $\mathrm{H}$, et al. circHIPK3 regulates lung fibroblast-to-myofibroblast transition by functioning as a competing endogenous RNA. Cell Death Dis. 2019; 10(3):182.

18. Ni H, Li W, Zhuge $\mathrm{Y}$, et al. Inhibition of circHIPK3 prevents angiotensin II-induced cardiac fibrosis by sponging miR-29b-3p. Int J Cardiol. 2019; pii: S0167-5273(18)37387-X

19. Li $\mathrm{Y}$, Zheng $\mathrm{F}$, Xiao $\mathrm{X}$, et al. CircHIPK3 sponges miR-558 to suppress heparanase expression in bladder cancer cells. EMBO Rep. 2017; 18(9):1646-1659.

20. Li B, Xie D, Zhang H. Long non-coding RNA GHET1 contributes to chemotherapeutic resistance to Gemcitabine in bladder cancer. Cancer Chemother Pharmacol. 2019; 84(1):187-194.

21. Xie D, Zhang H, Shang C. Long non-coding RNA CDKN2B antisense RNA 1 gene inhibits Gemcitabine sensitivity in bladder urothelial carcinoma. J Cancer. 2018; 9(12):2160-2166.

22. Li Y, Wan B, Liu L, Zhou L, Zeng Q. Circular RNA circMTO1 suppresses bladder cancer metastasis by sponging miR-221 and inhibiting epithelial-to-mesenchymal transition. Biochem Biophys Res Commun. 2019; 508(4):991-996

23. Lu Q, Liu T, Feng H, et al. Circular RNA circSLC8A1 acts as a sponge of $\mathrm{miR}-130 \mathrm{~b} / \mathrm{miR}-494$ in suppressing bladder cancer progression via regulating PTEN. Mol Cancer. 2019: 18(1):111.

24. Chi BJ, Zhao DM, Liu L, et al. Downregulation of hsa_circ_0000285 serves as a prognostic biomarker for bladder cancer and is involved in cisplatin resistance. Neoplasma. 2019; 66(2):197-202.

25. Jin $\mathrm{P}$, Huang $\mathrm{Y}, \mathrm{Zhu} \mathrm{P}$, et al. CircRNA circHIPK3 serves as a prognostic marker to promote glioma progression by regulating miR-654/IGF2BP3 signaling. Biochem Biophys Res Commun. 2018; 503(3):1570-1574.

26. Huang $X$, Li Z, Zhang $Q$, et al. Circular RNA AKT3 upregulates PIK3R1 to enhance cisplatin resistance in gastric cancer via miR-198 suppression. Mol Cancer. 2019; 18(1):71.

27. Shang J, Chen WM, Wang $\mathrm{ZH}$, et al. CircPAN3 mediates drug resistance in acute myeloid leukemia through the miR-153-5p/miR-183-5p-XIAP axis. Exp Hematol. 2019; 70:42-54.e3.

28. $\mathrm{Yu} \mathrm{H}$, Chen $\mathrm{Y}$, Jiang P. Circular RNA HIPK3 exerts oncogenic properties through suppression of miR-124 in lung cancer. Biochem Biophys Res Commun. 2018; 506(3):455-462.

29. Kai D, Yannian L, Yitian C, et al. Circular RNA HIPK3 promotes gallbladder cancer cell growth by sponging microRNA-124. Biochem Biophys Res Commun. 2018; 503(2):863-869.

30. Chen G, Shi Y, Liu M, Sun J. circHIPK3 regulates cell proliferation and migration by sponging miR-124 and regulating AQP3 expression in hepatocellular carcinoma. Cell Death Dis. 2018; 9(2):175.

31. Li C, Zhao Z, Zhou Z, Liu R. Linc-ROR confers gemcitabine resistance to pancreatic cancer cells via inducing autophagy and modulating the miR-124/PTBP1/PKM2 axis. Cancer Chemother Pharmacol. 2016; 78(6):1199-1207. 